\title{
Analysis on Phase Space Reconstruction and Chaotic Characteristic of Element Concentration Series of Gold Deposit
}

\author{
L. Wan \\ School of Mathematics and Information Science, \\ Guangzhou University \\ Key Laboratory of Mathematics and Interdisciplinary \\ Sciences of Guangdong Higher Education Institutes, \\ Guangzhou University \\ Guangzhou, China \\ *Corresponding author
}

\author{
Y.Q. Zhu \\ 1School of Mathematics and Information Science, \\ Guangzhou University \\ Guangzhou, China
}

\author{
X.C. Deng \\ 2Key Laboratory of Mathematics and Interdisciplinary Sciences of Guangdong Higher Education Institutes, Guangzhou \\ University \\ Guangzhou, China
}

\begin{abstract}
In this paper, the technique of phase space reconstruction and chaos theory are applied to study the chaotic characteristics of gold concentration from the continuous channel and drill cores in Dayingezhuang Deposit, Jiaodong Gold Province, China. The results show that the largest Lyapunov exponents are above zero, and correlation fractal dimensions are non-integer, showing the chaotic characteristics in the gold enrichment processes. The gold concentration series of drill cores exhibits stronger chaotic characteristics than channel, showing a more complex and uncertain behaviour in drill cores. By quantifying the chaotic characteristics of the ore-forming elements' series of a typical deposit, this study can provide a new method for the identification of mineralization intensity and scientific foundation for further determination of the extent of deposit concentration and delineation of the target mineralization zone.
\end{abstract}

Keywords-chaotic; gold concentration series; mineralization intensity; correlation dimension; largest Lyapunov exponent

\section{INTRODUCTION}

In geology, mineralization is a natural geo-process that involves the interaction of fluids with rocks in the earth crust in a specific geological environment. Recent studies have shown that the mineralization processes often are complex system occurring in an anomalous environment [1, 2]. Identification of the anomalous extent or the influenced domain of the complex mineralization system is essential for mineral exploration [3, 4]. The metallogenic elements' concentration of a deposit in its mineralization zone invariably shows a highly anomalous pattern that is suitable for nonlinear analysis $[5,6]$.

In recent years, non-linear study of the geological and geochemical data is mainly focusing on fractal or multifractal.
However, the ore-forming process might be better understood through chaotic analysis that belongs to nonlinear deterministic model [2]. Therefore, investigation in the oreforming process with solid phase and the effect of dynamics has great significance. Chaos theory is an important branch of nonlinear analysis, and is widely applied in the last few decades in a variety of systems, e.g., mechanical, chemical, physical, as well as in social sciences [7-10]. In particular, chaotic techniques, developed to extract qualitative and quantitative information from time series, have been applied recently to the study of a large variety of irregular, erratic signals and by now have demonstrated to can be very useful to reveal deep dynamical features [11].

A disseminated-veinlet deposit controlled by a large-scale fault often contains large quantities of ore and metal, yet each orebody has a complex geometry and irregular element distribution patterns in the alteration zones. In this paper, the gold concentration series of along drifts and drill cores were selected form Dayingezhuang Deposit, Jiaodong Gold Province, China, using the nonlinear analysis method to verify that gold concentration series are deterministic chaotic phenomena, which is to investigate the dynamical behaviour of gold concentration series or the influenced domain of the complex mineralization system.

\section{MATERIALS AND METHODS}

\section{A. Data acquisition and Descriptive Statistics}

The Dayingezhuang ore deposit is located in the middle segment of the Zhaoping fault zone in Jiaodong gold province, China. The Jiaodong gold province is famous for its gold production, and structure-controlled alteration rock gold 
deposits formed in the Mesozoic dominate the province, where the pyrite-sericite-quartz altered rock (pay rock) is distributed through the whole mineralized zone. And the gold ore deposit is with $3000 \mathrm{~m}$ in length, $30 \mathrm{~m} \sim 140 \mathrm{~m}$ in width; moreover, ore bodies of altered rocks extend like sloping wave in a strike and in trend. The reserves of the Dayingezhuang are more than 100 $\mathrm{t}$, with an estimated annual production greater than $2.6 \mathrm{t}$ [12$14]$.

The data of gold concentrations are obtained from the continuous drill cores sample and channel sample with $1 \mathrm{~m}$ length on $-290 \mathrm{~m}$ levels, and the lengths of data are greater than $100 \mathrm{~m}$. These samples were assayed. The results are used for reserve calculation as well as for this study. The gold orebody described in this study is delimited based on a cutoff concentration of $2 \mathrm{~g} / \mathrm{t}$.

\section{B. Analysis Methods of Chaos Characteristics}

1) Phase space reconstruction: Given a time series $\left\{x_{i}\right\}$, where $i=1,2, \cdots, N$, the phase space can be reconstructed using the method of the standard delay-coordinate embedding. In this higher dimension space, the vectors of phase space reconstruction can be expressed as below:

$$
X\left(t_{i}\right)=\left[x\left(t_{i}\right), x\left(t_{i}+\tau\right), \cdots, x\left(t_{i}+(m-1) \tau\right], \quad i=1,2, \cdots, M\right.
$$

where $\tau$ is the time delay, $m$ is the embedded dimension, i.e. the coordinate number of the phase space, and the constants $m$, $N, M$ and $\tau$ are related as $M=N-(m-1) \tau$. The dynamic properties of systems could be studied by reconstruction of the phase space if $m \geqq 2 D_{2}+1$, where $D_{2}$ is the fractal dimension of the system[15]. The reconstructed phase space and original phase space are diffeomorphically equivalent. Therefore, we can investigate the original phase space through studying the reconstructed phase space.

In order to reconstruct the attractor, estimation of the embedding dimension $\mathrm{m}$ and the embedding delay $\tau$ are essential. The method of autocorrelation function was used for determination of $\tau$ as the delay causing the value of $(1-1 / e)$ its initial of the autocorrelation function[16]. The embedding dimensions $m$ were estimated using the Grassberger-Procaccia algorithm method proposed by Grassberger[17,18], which is suitable for shorter time series.

2) Correlation dimension: The correlation dimension is a measure of the attractor dimensionality. The correlation dimension is estimated from the correlation integral, which is the number of points in the phase space of dimension $m$ that are closer than $r$. The correlation integral is calculated with the Grassberger-Procaccia correlation sum

$$
C(r)=\frac{2}{N(N-1)} \sum_{1 \leq i \leq j \leq N} \theta\left(r-d_{i j}\right), r>0
$$

where $d_{i j}=\left\|X_{i}-X_{j}\right\|,\|\cdot\|$ is the maximum distance. $\theta\left(r-d_{i j}\right)$ is the Heaviside function, with $\theta\left(r-d_{i j}\right)=1$ for $r>d_{i j}$, and $\theta\left(r-d_{i j}\right)=0$ for $r \leq d_{i j} . N$ is the size of the data set. For small values of $r$, the correlation integral will increase with the power of the correlation dimension $D_{c}$ when $r$ increases, as follow:

$$
C(r) \propto r^{D_{2}}
$$

It is obvious that $\ln C(r)$ is linear with $\ln r$, and the calculating formula of correlation dimension can be expressed as

$$
D_{2}=\lim _{r \rightarrow 0} \frac{\ln C(r)}{\ln r}
$$

For different values of $r$, the least squares regression is introduced to calculate the points $(\ln r, \ln C(r))$ in the non-scale region, and the slope is considered as the correlation dimension [17].

3) Lyapunov exponent: Another technique to determine the presence of chaotic behaviour is the largest Lyapunov exponent, which measures the divergence of nearby trajectories. As the system evolves, the sum of a series of attractor point values (in each dimension) will converge or diverge.

The Lyapunov exponent $\lambda$ indicates the average speed of the track separation in the phase space, and it can reflect the changing of variables with time and the sensitivity of the initial conditions in the chaos dynamical system effectively. If the track is shrinking in the direction $\lambda<0$ and the movement is stable, the system is not sensitivity to the initial conditions. If the track is separating rapidly in the direction $\lambda>0$, the system is sensitive to the initial conditions. For the discrete system or the nonlinear time series, we only calculate the largest Lyapunov exponent $\lambda_{\max }$, which is an important indicator of the existence of chaos and the chaotic characteristic in dynamical systems. Thus, a positive Lyapunov exponent is a strong indicator of chaos. It is ensured that the time series has chaos if only the largest Lyapunov exponent larger than zero and it indicate the chaos degree of the system directly.

The method used in calculating the largest Lyapunov exponent is based on averaging the local divergence rates or the local Lyapunov exponents. Rosenstein et al. proposed a new method to calculate the largest Lyapunov exponent from an observed series, which is small data method[18]. The largest Lyapunov exponent, the definition is:

$$
\lambda_{\max }(i, k)=\frac{1}{k \Delta t(M-k)} \sum_{j=1}^{M-k} \ln \frac{d_{j}(i+k)}{d_{j}(i)}
$$


where $\Delta t$ is the sample period, $k$ is a constant, $M=N-(m-1) \tau, d_{j}(i)$ is the distance of the $j$ th couple nearest position after $i$ time steps pass.

That is

$$
d_{j}(0)=\min _{j}\left\|X_{j}-X_{\hat{j}}\right\|
$$

And

$$
d_{j}(i)=\min _{j}\left\|X_{j+i}-X_{\hat{j}+i}\right\|
$$

Here, the mean period $P$ is estimated as the reciprocal of the mean frequency of power spectrum.

\section{RESULTS AND DISCUSSION}

In order to reconstruct the original phase space, we first estimate reconstruction parameters, the delay time $\tau$ and embedding dimension $m$. We calculate both the autocorrelation function with time lags of $1-40 \mathrm{~m}$, and the cures have dropped to $(1-1 / e)$ of its initial value of the autocorrelation function, as indicated by red arrow in Fig.1. The delay time $\tau$ for the drill cores and channel samples is 2 and 3 , respectively.

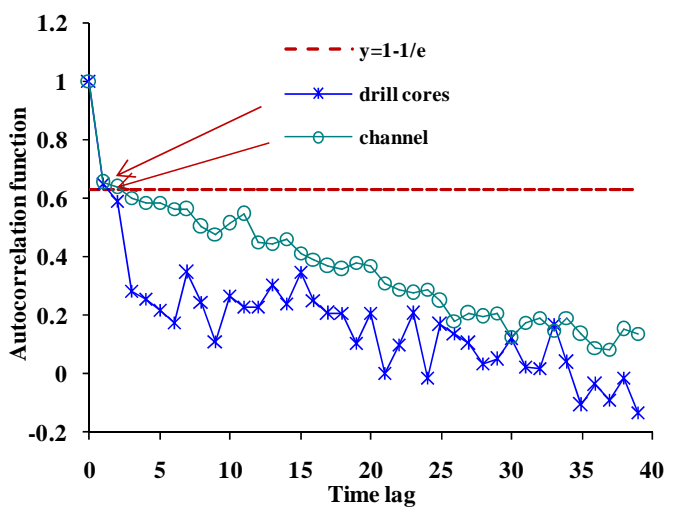

FIGURE I. AUTOCORRELATION FUNCTION OF THE GOLD CONCENTRATION SERIES

Subsequently, we calculate the embedding dimensions for our dataset using the method in the previous section 2.2.2, $\mathrm{m}$, from 2 to 26. Fig. 2(a)(b) shows the relationship between the correlation function $\ln C(r)$ and the radius $\ln r$ for increasing $m$, whereas the relationship between the correlation exponent values $D_{2}(m)$, and the embedding dimension values $m$, are shown by red in Fig. 3, $m=24$ and $D_{2}=3.961$ in gold concentration series of drill cores samples, $m=13$ and $D_{2}$ $=1.044$ in gold concentration series of channel samples (Fig.3). It can be seen that the correlation exponent value increases with the embedding dimension up to a certain value and then saturates beyond that value. The saturation of the correlation exponent beyond a certain embedding dimension value is an indication of the existence of deterministic dynamics.

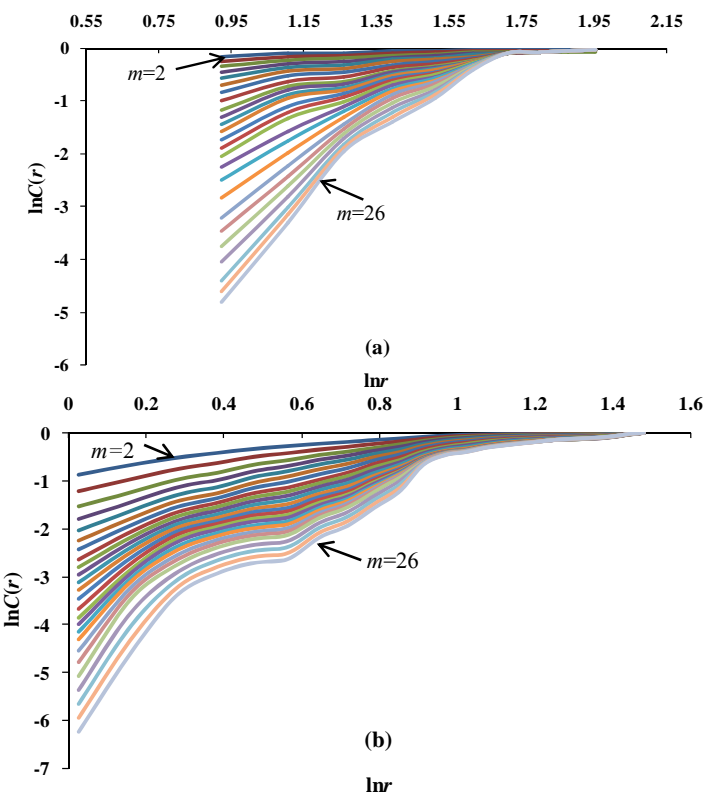

FIGURE II. $\ln C(r)$ VERSUS Ln $r$ PLOTS OF THE GOLD CONCENTRATION SERIES:(a) DRILL CORES SAMPLES; (b) CHANNEL SAMPLES

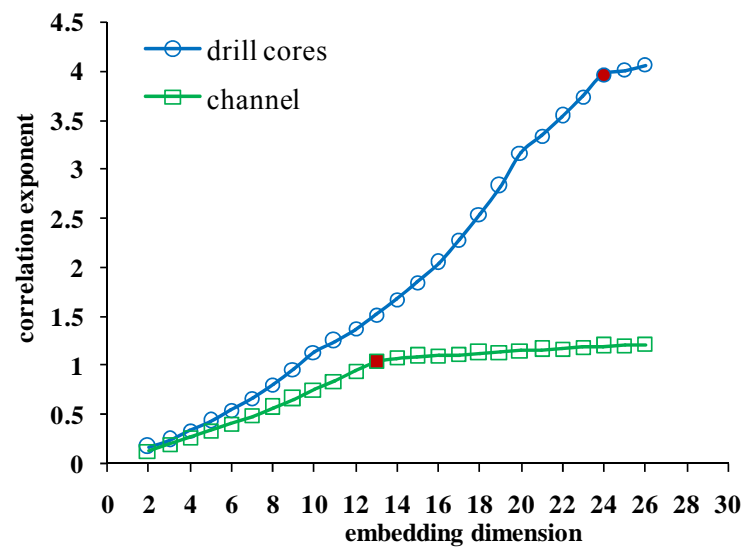

FIGURE III. RELATION BETWEEN CORRELATION EXPONENT $D_{2}(m)$ AND EMBEDDING DIMENSION $m$ : (a) DRILL CORES; (b) CHANNEL

The Lyapunov exponent are estimated using the small data sets method in section 2.2.3. The largest Lyapunov exponent is $\lambda_{\max }=0.0248,0.003>0$ for gold concentration of drill cores and channel sample, respectively. The result indicated the existence of chaotic characteristic at all of the four samples in mineralization processes. Moreover, by comparing of all the chaotic parameters, correlation dimension, embedding dimension and the positive largest Lyapunov exponent, we find that the chaotic parameters in channel sample is lower than drill cores sample, which indicates that the chaos level of the system in drill cores sample is more complex and uncertain behaviour.

\section{CONCLUSIONS}

The chaotic characteristics of the gold concentration series at the continuous channel samples and drill cores samples in 
Dayingezhuang Deposit, Jiaodong Gold Province, China, are investigated by the phase space reconstruction technology and chaos theory in this study. The results show that the largest Lyapunov exponents are above zero, and correlation fractal dimensions are non-integer, showing the chaotic characteristics in the gold enrichment processes. The study provides positive evidence regarding the existence of chaotic behaviour in metallogenic elements concentration series, leading to better understanding of the dynamics of the geology processes.

\section{ACKNOWLEDGEMENTS}

This research is supported by the National Natural Science Foundation of China (Grant No.41172295).

\section{REFERENCES}

[1] Cheng, Q.M. Mapping singularities with stream sediment geochemical data for prediction of undiscovered mineral deposits in Gejiu, Yunnan Province, China. ore geology reviews, 32, pp. 314-324, 2007.

[2] Yu, C.W. Fractal growth of mineral deposits at the edge of chaos. Education Press, Anhui, China, 2006.

[3] Agterberg, F.P. \& Cheng, Q.M. Multifractal modeling of fractures in the Lac Bonnet Batholith, Manitoba. computers \& geosciences, 22, pp. 97 $107,1996$.

[4] Cheng, Q.M. Quantifying the generalized self-similarity of spatial patterns for mineral resources assessment. Journal of Earth Science, 29, pp. 733-744, 2004.

[5] Turcotte, D.L. Fractals and chaos in geology and geophysics. Cambridge University Press, 1997.

[6] Turcotte, D.L. Fractals in petrology. Lithos, 65, pp. 261-271, 2002.

[7] Rawat, S., Saha, B., Prasad, A. \& Chandra, A. Chaotic behavior of ion exchange phenomena in polymer gel electrolytes through irradiated polymeric membrane. Physics Letters A, 376, pp. 1915-1918. 2012.

[8] Hacinliyan, A.S., Skarlatos, Y., Kusbeyzi Aybar, I., Aybar, O.O., Shpotyuk, O., Golovchak, R., Balitska, V. \& Kozdras, A. Natural physical aging in glassy As-Se: A comparative study of chaotic behavior with enhanced results analysis. Journal of Non-Crystalline Solids, 386 , pp. 8-13, 2014

[9] Remya, R. \& Unnikrishnan, K. Chaotic behaviour of interplanetary magnetic field under various geomagnetic conditions. Journal of Atmospheric and Solar-Terrestrial Physics, 72, pp. 662-675, 2010.

[10] Atin, D. \& Pritha, D. Chaotic analysis of the foreign exchange rates. Applied Mathematics and Computation, 185, pp. 388-396, 2007.

[11] Edward, O. Chaos in Dynamical Systems. Cambridge University Press, 2002.

[12] Deng, J., Wang, Q.F., Wan, L., Yang, L.Q., Gong, Q.J., Zhao, J. \& Liu, H. Self-similar fractal analysis of gold mineralization of Dayingezhuang disseminated-veinlet deposit in Jiaodong gold province, China. Journal of Geochemical Exploration, 102, pp. 95-102, 2009.

[13] Deng, J., Wang, Q.F., Wan, L., Liu, H., Yang, L.Q. \& Zhang, J. A multifractal analysis of mineralization characteristics of the Dayingezhuang disseminated-veinlet gold deposit in the Jiaodong gold province of China. Ore Geology Reviews, 40, pp. 54-64, 2011.

[14] Wan, L., Wang, Q.F., Deng, J., Gong, Q.J., Yang, L.Q. \& Liu, H. Identification of Mineral Intensity along Drifts in the Dayingezhuang Deposit, Jiaodong Gold Province, China. Resource Geology, 60(1), pp. 98-108, 2010.

[15] Takens, F. Detecting strange attractors in turbulence. Lecture Notes in Mathematics, 898, pp. 366-381. 1981.

[16] Liebert,W. \& Schuster H.G. Proper choice of the time delay for the analysis of chaotic timeseries, Physics Letters A 142, pp. 107-111, 1989.

[17] Grassberger, P. \& Procaccia, I. Measuring the strangeness of strange attractors. Physica D, 9, 189-208, 1983.
[18] Rosenstein, M.T., Collins, J.J. \& De luca, C.J. A practical method for calculating largest Lyapunov exponents from small data sets. Physica D, 65, pp. 117-134, 1993. 